\title{
Surgical outcome of cortical dysplasias presenting with chronic intractable epilepsy: A 10-year experience
}

\author{
Manjari Tripathi ${ }^{1}$, Mahendra S. Singh, M. V. Padma', Shailesh Gaikwad², C. S. Bal ${ }^{3}$, Madhavi Tripathi ${ }^{3}$, \\ C. Sarkar ${ }^{4}$, Aditya Gupta, Garima Shukla1, V. P. Singh, Satish Jain ${ }^{1}$, Bhawani S. Sharma, \\ P. Sarat Chandra
}

Departments of Neurosurgery, ${ }^{1}$ Neurology, ${ }^{2}$ Neuroradiology, ${ }^{3}$ Nuclear Medicine, ${ }^{4}$ Neuro-pathology, All India Institute of Medical Sciences (AlIMS), New Delhi, India.

\begin{abstract}
Background: There has been sparse description of cortical dysplasias (CDs) causing intractable epilepsy from India. Aim: Clinical retrospective study of CDs causing intractable epilepsy that underwent surgery. Materials and Methods: Fifty-seven cases of CDs reviewed (1995 till July 2006) are presented. All patients had intractable epilepsy, and underwent a complete epilepsy surgery workup (inter ictal electroencephalography (EEG), video EEG, MRI as per epilepsy protocol, SPECT \{interictal, ictal with subtraction and co-registration when required\}, and PET when necessary). Surgical treatment included a wide exposure of the pathology with a detailed electrocorticography under optimal anesthetic conditions. Mapping of the sensori-motor area was performed where indicated. Procedures included resection either alone or combined with multiple subpial transactions when extending into the eloquent areas. Results: Our study had 28 $(49.12 \%)$ cases of isolated focal CDs, and 29 (50.67\%) with dual pathology. Average age at the time of onset of seizures in our series was 7.04 years (three months to 24 years), and average age at the time of surgery was 10.97 years (eight months to 45 years). Among coexistent pathologies, one had associated MTS, 16 had coexistent gangliogliomas and 12 (dysembryonic neuroepithelial tumor) DNTs. At an average follow-up of 3.035 years (range 5-10 years), three patients were lost to follow-up. Fifty-one per cent (29/57) patients had a good outcome (Engel Grade I) and 26\%(15/57) had a Grade II outcome. Conclusion: Cortical dysplasias have a good outcome if evaluated and managed with concordant electrical and imaging modalities.
\end{abstract}

Key words: Cortical dysplasia, hemispherotomy, intractable epilepsy, outcome, surgery, India, developing country

Cortical dysplasia (CD) is an uncommon pathology causing intractable epilepsy. Over the recent years, more cases are being recognized with improvement in diagnostics and imaging. Since the inception of the epilepsy surgery program in 1995, we have operated upon more than 400 cases of intractable epilepsy at our center. Amongst these cases are 57 focal cortical dysplasias (FCDs). ${ }^{[1]}$

The results reported for FCD have been variable. ${ }^{[2-4]}$ Sisodya ${ }^{[5]}$ in his meta-analysis of all outcome studies of surgery for CDs (till 2000) described $40 \%$ seizure-free, or good outcome in cases of multiple CDs, focal CD being the most common variety. In comparison to temporal lobe epilepsy this is a lower outcome. However, latest studies indicate a better outcome. ${ }^{[6-9]}$ We present here our experience with this uncommon pathology for the last 10 years.

\section{Materials and Methods}

All patients since 1995 till July 2006, with proven CD (isolated or dual) were analyzed on the basis of discharge summaries, imaging records, operative notes and personal telephonic conversation. Since this was a retrospective clinical study, detailed consent forms were already obtained. Thirteen patients could not be included in this study as it was felt that the requisite data was not available for this study.

Fifty-seven consecutive patients of all age groups (range eight months to 45 years), with FCD were included in this study

The presurgical evaluation of potential candidates included a detailed history and clinical examination, interictal EEG, long-term video EEG and high-quality magnetic resonance imaging (MRI) scan (including SPGR, T1 and fluid attenuated inversion recovery (FLAIR) sequences in oblique coronal planes 3-mm thick, perpendicular to the principal axis of the hippocampal formation and sensitive enough to detect subtle alterations in hippocampal size, morphology and signal intensity). Gadolinium enhancement was used if a structural pathology was suspected. All patients underwent long-term video EEG monitoring and a 
minimum of three seizures were recorded. The patient was considered for surgery only if a concordance could be established with imaging. An interictal SPECT study was done using HMPAO. In doubtful cases, an ictal SPECT was done using Tc-ECD. Ictal and interictal subtraction (SISCOS) and co-registration with MRI (SISCOS) was performed where necessary. PET (with fluro deoxyglucose) was performed where indicated.

Once the concordance was confirmed, the patient was subjected to appropriate surgery. This included a wide craniotomy to expose the pathology. At surgery, in most of the instances, the abnormal pathology could be identified by gentle palpation with a wet gloved finger. In addition, in most of the cases there was presence of gyral anomalies like pachygyria or presence of paler tissues over the anomaly. The next step was to perform a detailed electrocorticography (ECOG). A 4x4 grid with platinum electrodes was generally preferred. However, for larger lesions, a 8x8 grid was used. During the ECOG the grids were positioned in different areas to map the abnormal activity. When the lesion was close to the motor or sensory cortex, SSEP was performed by stimulating the median nerve and the central sulcus was identified by noting the reversal of potentials.

Electrocorticography in the operating room was performed under standard anesthetic conditions. Care was also taken to avoid using any wireless equipment or use of unnecessary electrical equipment to avoid artifacts. After craniotomy and dural opening, patients were pharmacologically paralyzed, and anesthesia maintained with nitrous oxide and intravenous narcotics with discontinuation of other inhalation agents. Core body temperature was maintained above $35.5^{\circ} \mathrm{C}$. Electrocorticography was recorded when the end-tidal inhalation agent (by mass spectroscopy) was below $0.1 \%$ (sevoflurane or isoflurane). Reference and ground needle electrodes were placed over the contralateral frontal-central scalp. The low-frequency filter was set at $1 \mathrm{~Hz}$, the high-frequency filter at $70 \mathrm{~Hz}$, and sensitivity was between 20 and $50 \mathrm{mV} / \mathrm{cm}$. Multiple contact grid electrodes (24-28 contacts) were positioned over the exposed cortical surface, and the recording was performed over 15-20 min site. Electrocorticography abnormalities were graded using the following score. [20-22]

ECoG Score 1: Normal background of mixed gamma, beta and alpha frequencies of moderate to low amplitude (i.e., usually 20-30 mV). A few low-amplitude spikes could be observed.

ECoG Score 2: Loss of fast $(120 \mathrm{~Hz})$ background frequencies, but otherwise a background of mixed alpha, beta and delta frequencies of low to moderate amplitude. Repeated but non-continuous, spikes, polyspikes or paroxysmal fast activity of medium amplitude often observed.

ECoG Score 3: Mostly 6-20 Hz background frequencies with some localized nearly continuous interictal epileptiform features of moderate amplitude or persistent repetitive spiking. Very rarely, electrographic seizures were captured.

ECoG Score 4: Slow (16 Hz) background frequencies with continuous synchronous features of moderate to high amplitude. Multiple independent epileptiform abnormalities (polyspikes, paroxysmal fast activity and electrographic seizures) could be recorded.

ECoG Score 5: Slow rhythmic usually synchronous background $(14 \mathrm{~Hz})$, often of high amplitude. Continuous synchronized or independent highamplitude epileptiform abnormalities in multiple cortical sites could be observed. Ictal discharges were rarely recorded but observed in surrounding cortex.

An ECoG score of 2-5 was considered abnormal and area showing this activity was always included in the resective area. An ECoG Score 1 was considered relevant depending on the existing situation. For instance, if there was widespread hemispheric activity consistent with ECoG Score 1 activity, then 'chasing the spikes' was avoided, and the maximally abnormal area was resected. If this activity was more focal amenable to surgical resection, then it was utilized for guiding surgical resection.

Surgery included a wide resection, making sure that the subcortical white matter was also included in the resection. A subpial transaction was performed in addition, if the ECOG showed abnormal activity over the motor or sensory cortex. One patient had a hemispheric CD and underwent a hemispherotomy. All resected specimens were subjected to a complete histopathological examination, and grading as per Palmini's classification was performed in 31 cases. A post-resection ECoG was always performed. If significant abnormal activity was still noted from the margins, further resection was performed.

Following surgery, patients were continued on antiepileptic drugs (AED) and the scheduled drug dosage was not omitted either during surgery or the same day. If the patient was receiving phenytoin or valproate, a bolus injection was given at the time of the surgery. If he/she was receiving an AED with no injectable form available, then a double dose was given in the morning through a Ryele's tube which was then removed. The same was repeated at night after surgery.

The follow-up was obtained using patient's OPD records, and personal telephonic conversation. The outcome was classified using Engel grade ${ }^{[17]}$ (Grades I-1V, where Grade I represented being completely seizure-free and was included in good outcome).

The outcome, the histopathological grades and other demographic factors were correlated with each other.

Focal cortical dysplasias were histologically classified on the basis of Palmini's grading. ${ }^{[3]}$ In this 
grading system, two types of dysplasias are identified based on the presence or absence of dysmorphic neurons or balloon cells. In Type I FCD, there are no dysmorphic neurons or balloon cells. In Type IA, isolated architectural abnormalities, usually laminar or columnar disorganization, are found. Type IB is also characterized by architectural abnormalities, but giant cells or immature neurons are also seen. It is important to note that no abnormal cells are present in Type I FCD. On the other hand, abnormal neurons are found in Type II FCD. In Type IIA, there are dysmorphic cells but no balloon cells. In Type IIB, both dysmorphic cells and balloon cells are found.

\section{Results}

\section{Age and sex distribution}

The average age at the time of onset of seizures was $7.04(+6.24)$ years (range three months-24 years). Average age at the time of surgery was $10.97(+8.12)$ years (range eight months- 45 years). However, at the time of surgery, the majority of patients (35/57 - 61.4\%) were under 18 years of age.

Among these patients, there were 33 males, with a sex ratio of 1.375. All cases had confirmed histopathology of CD with or without coexisting pathologies [Table 1].

Investigations: All patients underwent interictal EEG, VEEG, MRI as per epilepsy protocol. In the more recent years, 3-T MRI was performed when indicated (11 cases). SPECT using HMPAO was performed in all 57 cases. Ictal SPECT was performed in 32 cases. Subtraction imaging (using SISCOS or SISCOM) was performed in 29 cases. PET (with fluro-dexoyglucose) as additional investigation was performed in 13 cases.

Clinical characteristics: All the patients had 'intractable epilepsy' in that they still continued having seizures despite being on two on more AEDs for two or more years (non-epileptic events and poor compliance being ruled out). Eleven patients presented with catastrophic seizures and status and were not responding to anticonvulsants. The average seizure frequency was 2.8 / week (+ 1.9- 2 S.D). The range varied from status converting to 5-6 seizures a day to 3-4 seizures a month . The seizure semiology included complex partial seizures in a majority of patients $(\mathrm{n}=32 ; 56.14 \%)$, followed by simple partial seizures $(\mathrm{n}=21 ; 36.8 \%)$.
About eight patients had infantile spasms. Even though a formal neuropsychological testing was not done in all cases (done in only 19 cases), evaluation of the case records showed presence of cognitive delay in 13 cases (seven in cases where a detailed neuro-pyschological testing was done)

\section{Isolated CDs and coexisting pathologies}

Of the 57 cases reported here, 28 had isolated CDs, while 29 had a coexisting independent pathology (ganglioglioma $\mathrm{n}=16$, dysembryoplastic neuroepithelial tumor [DNT] $\mathrm{n}=12$, mesial temporal sclerosis [MTS] $\mathrm{n}$ $=1$ ) [Table 2]. In the case of MTS, the FCD was placed in the medial temporal region.

\section{Lobar distribution}

The lobar distribution was assessed on the basis of imaging and electrophysiological studies [Table 1]. One patient had a hemispheric CD requiring hemispherotomy.

\section{Follow-up}

The average duration of follow-up was $3.035(+5.8)$ years with a range of five months to 10 years. Three patients were lost during follow up and one patient was lost after a follow up of five months.

\section{Outcome}

Good outcome was measured in terms of Engel's grades I- IV. Distribution of patients in terms of Engel's grade was as follows:

$\begin{array}{llc}\text { - } & \text { Grade I } & 29 \\ \text { - } & \text { Grade II } & 15 \\ \text { - } & \text { Grade III } & 8 \\ \text { - } & \text { Grade IV } & 3\end{array}$

Hence good outcome (Grade I) was seen in 51\%. Engel's grade was not available for three patients as they were lost to follow-up, and one patient was Grade IV at five months, but was lost to follow-up after five months.

In our series, no patient undrewent isolated multiple subpial transactions (MST) as in all cases the FCD was such that it was primarily present over a relatively noneloquent cortex extending into an eloquent zone. Hence an MST was always combined with a resection. There

\begin{tabular}{|c|c|c|c|}
\hline & Frontal & Temporal & $\begin{array}{l}\text { More than } 1 \text { lobe } \\
\text { (fronto-parietal 17) }\end{array}$ \\
\hline Location (coexisting independent pathology in parenthesis) & $7(3)$ & $21(12)$ & $29(14)$ \\
\hline Mean Age $(+6.24)$ of onset of seizures (Range, no. of males) & $6.51(0.6-22,4)$ & $8.20(4.9-16,14)$ & $6.41(4.8-45,15)$ \\
\hline Coexisting Pathology & 1 (GG), 4(DNT) & 11(GG), 6(DNT), 1(MTS) & 4(GG), 2 (DNT) \\
\hline Good outcome (Engel's Grade I) & $\begin{array}{l}\text { (CD:2,+GG: } 1, \\
\text { +DNT:0): Total: } 3\end{array}$ & $\begin{array}{c}\text { (CD:11, +GG:9, } \\
\text { +DNT:2, +MTS:0). Total: } 22\end{array}$ & $\begin{array}{l}\text { (CD: } 3,+ \text { +GG:1, } \\
\text { +DNT:0). Total: } 4\end{array}$ \\
\hline
\end{tabular}

CD: Cortical dysplasia, +GG: Ganglioglioma + CD, DNT: Dysembryonic neuroepithelial tumor + CD, (Some patients had overlapping localization e.g. frontal as well as parietal) 
was no difference in outcome between patients who had resection vs. a combination of resection combined with MST.

Keeping in mind that a significant number of pathologies were dual, the outcome analysis was performed for isolated CD vs. dual pathologies. This revealed a good outcome in about 61\% (Grade I) for isolated CDs while the overall seizure-free outcome (Engel Grade I) for dual pathologies is around $46 \%$. We do not know the cause for this discrepancy in outcome. However, one of the possibilities is that the diagnosis of CD came as a surprise and it was only the other pathology which was considered as the primary pathology, hence it is always possible that some residual dysplastic tissue may have been left behind accounting for a lower seizure-free outcome. Even though ECoG was always performed to guide the extent of resection, it is possible that the area with FCD was electrically silent in the presence of another pathology and could have become active once the primary pathology was excised [Table 2]. The only case of CD associated with mesial temporal sclerosis (MTS) had a Grade IV outcome. In this case, the preoperative diagnosis was MTS only. The diagnosis of CD was made only after the histopathological examination, hence it is possible that some residual CD left behind may be responsible for the poor outcome. This patient was scheduled for a repeat MRI but was lost to follow-up.

The outcome with respect to Palmini's grading is shown in Table 3.Thus it is evident that out of 31 patients whose slides were reviewed for grading, 22 (70.96\%) patients had a Grade I outcome. Of these maximum improvement was seen in cases with Palmini's grade IIb (100\%) followed by Palmini Grade IIa (83.3\%).

Table 2: Seizure outcome of isolated CD vs. dual pathologies. GG; ganglioglioma, DNT: dysembryonic neuroepithelial tumor, MTS: mesial temporal sclerosis

\begin{tabular}{lcccc}
\hline Engle grade & CD & CD+GG & CD+DNT & CD+MTS \\
Grade I & $16^{*}$ & 11 & 2 & 0 \\
Grade II & $5^{*}$ & 4 & 6 & 0 \\
Grade III & 3 & 1 & 4 & 0 \\
Grade IV & 2 & 0 & 0 & $1^{*}$ \\
Total & $26^{*}$ & 16 & 12 & 1 \\
\hline
\end{tabular}

*Three patients were lost to follow-up

\begin{tabular}{lccc}
\hline \multicolumn{2}{c}{ Table 3: Seizure outcome compared with Palmini's grading } \\
& \multicolumn{2}{c}{ (Palmini's grading done for 31 cases only) } \\
\hline Grade & Total & $\begin{array}{c}\text { Grade I Engel's } \\
\text { grading }\end{array}$ & $\begin{array}{c}\text { Grades II-IV Engel's } \\
\text { grading }\end{array}$ \\
la & & $4(66.66)$ & $2(33.33)$ \\
Ib & 11 & $6(54.54)$ & $5(45.45)$ \\
lla & 12 & $10(83.33)$ & $2(16.66)$ \\
llb & 2 & $2(100)$ & 0 \\
Total & 31 & $22(70.96)$ & $9(29.03)$ \\
\hline
\end{tabular}

Figures in parentheses are in percentage

\section{Postoperative complications}

Three patients had meningitis, proven by lumbar puncture and these were treated appropriately. Two patients had hemiparesis and both showed gradual recovery at subsequent follow-up. One of these patients also had a contralateral superior quadrantanopia, which gradually improved to near normal. Both these patients had a large CD extending close to motor strip. In one case, a male child eight years old, had extensive bi-occipital CDs [Figure 1]. Ictal SPECT localized the epileptic zone on the right occipital lobe but electrophysiological findings were bilateral. He underwent a bilateral occipital craniotomy. Intraoperative ECOG showed significant activity from the right lateral occipital lobe with some activity also coming from the left side. He thus underwent right-sided resection and a MST on the left side. Following surgery, this patient developed status epilepticus which required him to be paralyzed and ventilated for $48 \mathrm{~h}$. He improved subsequently to have an Engel's Grade II outcome. The overall complication rate was $9 \%$ with only one patient having a significant residual hemiparesis. There was no mortality in our series.

\section{Discussion}

Background: The term "cerebral cortical dysplasia" encompasses a spectrum of malformations of the cerebral cortex that arise during development and are associated with epilepsy in infants and children, ${ }^{[10]}$ though they may be also seen in older patients with longstanding epilepsy, particularly in developing countries. Initially, cortical malformations were referred to largely by their gross characteristics (e.g., lissencephaly, agyria or pachygyria, hemimegalencephaly, microgyria). As investigators discovered the range of microscopic cortical malformations that produce epilepsy but show no (or milder) gross abnormalities, additional terms such as "microdysgenesis",[11] "dysplastic cortical architecture", ${ }^{[12]}$ "focal cortical dysplasia", ${ }^{[2]}$ were added

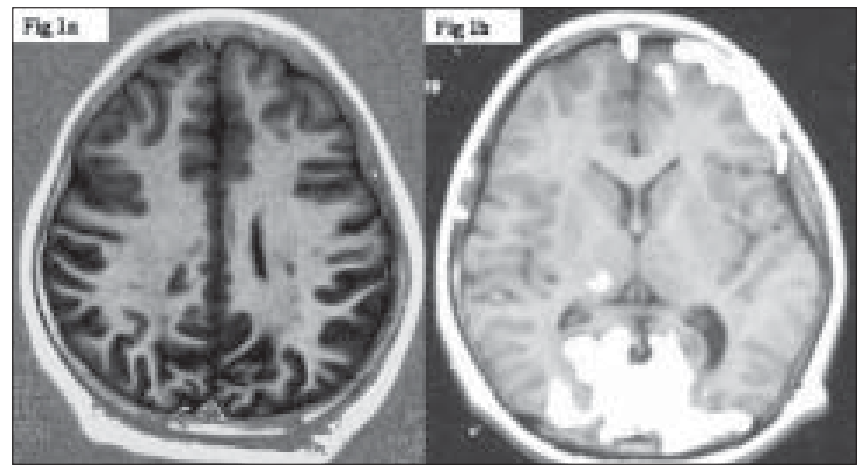

Figure 1: A nine-year-old boy presented with complex partial becoming generalized epilepsy, MRI showing [Figure 1a] bilateral occipital cortical dysplasias. Functional MRI using visual stimulation showed that both the occipital lobes were active [Figure 1b] 
to the literature. The term "focal cortical dysplasia" was first used to describe a specific malformation of the brain that consisted of disorganized cortex with enlarged irregular neurons and enlarged ballooned cells. ${ }^{[2]}$

The clinical form, age at onset of symptoms, and severity of epilepsy that a given patient with CD manifests appear to depend most on cerebral embryogenesis. ${ }^{[11]}$ In general, the more severe the CD, the earlier the onset of symptoms and the more severe the epileptic syndrome. ${ }^{[3-10]}$

The past decade has witnessed a large number of clinical and laboratory-based studies related to FCD[13-22] (to quote a few). While these pathologies were more difficult to diagnose especially on low resolution MRI's. However development of more contemporary MR software and imaging technologies like FLAIR, SPGR, diffusion-weighted imaging ${ }^{[15,19]}$ etc have greatly enhanced the diagnostic capabilities. Even in our series 18 patients had undergone an MRI elsewhere which was reported as normal.

Study question: Despite a large body of literature available, very few studies have been quoted from India, most of them being part of larger series of epilepsy surgery, ${ }^{[23-25,27]}$ including from our center earlier. This was one of the main reasons which encouraged updating and analyzing our results of FCD separately to evaluate the various clinical and pathological parameters. The present series is thus a large compilation of a decade of experience of a relatively uncommon pathology causing epilepsy.

However, the main shortcoming of this series is that it is a retrospective study, this being the main reason why 13 patients could not be included in this study due to non-availability of complete data.

Epilepsy was the most common presenting symptom in our series too . In our series, $54.38 \%$ of patients i.e. 31 patients were at or below five years of age at the time of presentation. Commencement of seizures below 10 years of age was seen in 45 i.e. $78.9 \%$ patients.

However, our study was unusual in that a very significant number of cases $(49.1 \%, 28 / 57$ patients) had coexisting pathologies in the form of DNT (12) and gangliogliomas (16) and MTS (1) as compared to earlier studies. ${ }^{[20-24]}$ In all these cases, the diagnosis of CD was made only after histopathological examination. More so the seizure-free outcome (Grade I Engel) was much lower (46\% vs. 61\%) in patients who had a double pathology. We do not know this reason .

In our earlier study, where we analyzed our histopathological data of our resective epilepsy surgery cases ${ }^{[1]}$ the commonest cause of a surgical substrate causing intractable epilepsy was MTS (25\%), followed by tumors (20\%); FCD accounted (including those with dual pathologies) for $15 \%$.

Neoplastic pathologies like GG and DNET have been observed in association with FCD; although the nature of this remains controversial. ${ }^{[20-32]}$ Whether these neoplasms arise from FCD, represent some peculiar form of dysplasia, or merely coexist with dysplasia remains to be ascertained. It has been suggested that ${ }^{[33-}$ ${ }^{35]}$ associated glioneuronal neoplastic and malformative lesions are associated with CD-34-immunopositive bipotent precursor cells. However, all these series have extended histopathological studies to the ultrastructural level that was not done in our study.

The good outcome in our series (51\%) seems to be comparable to what has been usually quoted in the literature. ${ }^{[2-19]}$ However, we do submit the lacunae of the present study. Firstly it is a retrospective study. Secondly, our follow-up even though reasonable (about three years mean) is still small considering the natural history of these pathologies following a surgical intervention.

None of our patients ever required invasive video EEG. This could be partly due to the fact that all our patients underwent a very exhaustive investigatory workup (EEG, MRI with special sequences, ictal and interictal SPECT with SISCOS and SISCOM, and PET). As many as 18 patients had undergone a 'routine' MRI elsewhere which was diagnosed as normal before being worked up at our institute, thus the pick-up and localization has been quite accurate which is also likely to translate into a good outcome. Furthermore, all our patients have had electrical and imaging concordance thus allowing for direct resective surgery after ECoG evaluation at surgery. However, we do have some patients on our follow-up without surgery who have FCD without electrical and imaging concordance. We are planning for an invasive video EEG for these patients.

\section{Conclusions}

The following series has been a clinico-pathological experience of FCDs collected over a 10-year period. This, we believe is the first of its kind of study from India and demonstrates that surgery for these pathologies is associated with low morbidity and seizure-free outcome (Grade I) in 51\% of the operated cases, at a mean follow-up of three years. However, a longer follow-up would be required to actually comment on the natural history. While most of the results in this study have been similar to others, an interesting aspect of this study has been a higher incidence of coexisting pathologies (50\%). In future, we plan to perform a more detailed cytomolecular analysis including patch clamp techniques, particularly to compare between adult and pediatric FCDs.

Ethical clearance: The study involved principles of treatment well established in the literature, hence did not merit an ethical clearance. 


\section{Acknowledgment}

The authors wish to thank Ms Prarthana for helping in the preparation of the manuscript.

\section{References}

1. Sarkar C, Sharma MC, Deb P, Singh VP, Chandra PS, Tripathi M, \& al. Neuropathological spectrum of lesions associated with intractable epilepsies: A 10-year experience with a series of 153 resections. Neurol India 2006;54:144-50.

2. Taylor DC, Falconer MA, Bruton CJ, Corsellis JA. Focal dysplasia of the cerebral cortex in epilepsy. J Neurol Neurosurg Psychiatry 1971;34: 369-87.

3. Palmini A, Andermann F, Olivier A, Tampieri D, Robitaille Y, Andermann E, et al. Focal neuronal migration disorders and intractable partial epilepsy: A study of 30 patients. Ann Neurol 1991;30:741-9.

4. Raymond AA, Fish DR, Sisodiya SM, Alsanjari N, Stevens M, Shorvon SD. Abnormalities of gyration, heterotopias, tuberous sclerosis, focal cortical dysplasia, microdysgenesis, dysembryoplastic neuroepithelial tumor, and dysgenesis of the archicortex in epilepsy. Brain 1995;118:629-60.

5. Sisodiya SM. Surgery for malformation of cortical development causing epilepsy. Brain 2000;123:1075-91.

6. Kloss S, Pieper T, Pannek H, Holthausen H, Tuxhorn I. Epilepsy surgery in children with focal cortical dysplasia (FCD): Results of long-term seizure outcome. Neuropediatries 2002;33:21-6.

7. Cohen-Gadol AA, Orduman K, Bronen RA, Kim JH, Spencer DD. Long-term outcome after epilepsy surgery for focal cortical dysplasia. J Neurosurg 2004;101:55-65.

8. Chung CK, Lee SK, Kim KJ. Surgical outcome of epilepsy caused by cortical dysplasia. Epilepsia 2005;46:S25-9.

9. Prayson RA, Estes ML, Morris HH. Co Existence of neoplasia and CD in patients presenting with seizures. Epilepsia 1993;34:609-15.

10. Mischel PS, Nguyen L, Vinters HV. Cerebral cortical dysplasia associated with pediatric epilepsy: Review of neuropathologic features and proposal for a grading system. J Neuropathol Exp Neurol 1995;54:137-53.

11. Meencke HJ, Janz D. Neuropathological findings in primary generalized epilepsy: A study of eight cases. Epilepsia 1984;25:8-21.

12. Rorke LB. A perspective: The role of disordered genetic control of neurogenesis in the pathogenesis of migration disorders. J Neuropathol Exp Neurol 1994;53:105-17.

13. Chevassus-Au-Louis N, Congar P, Represa A, Ben-Ari Y, Gaiarsa JL. Neuronal migration disorders: Heterotopic neocortical neurons in CA1 provide a bridge between the hippocampus and the neocortex. Proc Natl Acad Sci USA 1998;95:10263-8.

14. Clark GD, MeNeil RS, Bix GJ, Swann JW. Platelet-activating factor produces neuronal growth cone collapse. Neuroreport 1995;6:2569-75.

15. Colombo N, Citterio A, Galli C, Tassi L, Lo Russo G, Scialfa G, \& al. Neuroimaging of focal cortical dysplasia: Neuropahologic correlations. Epileptic Disord 2003;5:S67-72.

16. Kuzniecky R. Familial diffuse cortical dysplasia. Arch Neurol 1994;51:307-10.

17. Vinters HV, Armstrong DL, Babb TL, \& al. The neuropathology of human symptomatic epilepsy. In: Engel J Jr, editor. Surgical treatment of the epilepsies. 2nd ed. New York: Raven; 1993a. p. 593-608.

18. Zilles K, Qu M, Schleicher A, Luhmann HJ. Characterization of neuronal migration disorders in neocortical structures: Quantitative receptor autoradiography of ionotropic glutamate, $\mathrm{GABA}(\mathrm{A})$ and $\mathrm{GABA}(\mathrm{B})$ receptors. Eur J Neurosci 1998;10:3095-106.

19. Chandra PS, Salamon N, Huang J, Wu JY, Koh S, Vinters HV, \& al. FDG-PET/MRI coregistration and diffusion-tensor imaging distinguish epileptogenic tubers and cortex in patients with tuberous sclerosis complex: A preliminary report. Epilepsia 2006;47:1543-9.

20. Cepeda C, Andre VM, Levine MS, Salamon N, Vinters HV, Mathern GW, etal. Epileptogenesis in pediatric cortical dysplasia: The dysmature cerebral developmental hypothesis. Epilepsy Behav 2006; 9:219-35.

21. Cepeda C, Andre VM, Vinters HV, Levine MS, Mathern GW. Are cytomegalic neurons and balloon cells generators of epileptic activity in pediatric cortical dysplasia? Epilepsia 2005;46:S82-8.

22. Cepeda C, Andre VM, Flores-Hernandez J, Nguyen OK, Wu N, Klapstein GJ, \& al. Pediatric cortical dysplasia: Correlations between neuroimaging, electrophysiology and location of cytomegalic neurons and balloon cells and glutamate-GABA synaptic circuits. Dev Neurosci $2005 ; 27: 59-76$.

23. Sylaja PN, Radhakrishnan K, Kesavadas C, Sarma PS. Seizure outcome after anterior temporal lobectomy and its predictors in patients with apparent temporal lobe epilepsy and normal MRI. Epilepsia 2005; $46: 600$.

24. Bhatia M, Singh VP, Jain S, Gaekwad S, Bal CS, Sarkar C, đal. Epilepsy surgery in India: All India Institute of Medical Sciences experience. J Assoc Physicians India 1999;47:492-5.

25. Shukla G, Bhatia M, Singh VP, Tripathi M. Successful selection of patients with intractable extratemporal epilepsy using non-invasive investigations. Seizure 2003;12:573-6.

26. Palmini A, Gambardella A, Andermann F, Dubeau F, da Costa JC, Olivier A, \&al. Operative strategies for patients with cortical dysplastic lesions and intractable epilepsy. Epilepsia 1994;35:S57-71.

27. Carlos S. Invited comments. Neurol India 2006;54:150-1.

28. Engel J Jr. Outcome with respect to epileptic seizures. In: Engel J Jr editor. Surgi treatment of the epilepsies. New York: Raven Press; 1987.

29. Prayson RA, Frater JL. Cortical dysplasia in extratemporal lobe intractable epilepsy: a study of 52 cases. Ann Diagn Pathol 2003;7:13946.

30. Frater JL, Prayson RA, Morris HH 3rd, Bingaman WE. Surgical pathologic findings of extratemporal based intractable epilepsy. Arch Pathol Lab Med 2000;124:545.

31. Wolf HK, Muller MZ, Spanle M, Zentner J, Schramm J, Wiestler OD, Ganglioglioma: A detailed histopathological and immunohistochemical analysis of 61 cases. Acta Neuropathol 1994;88:166-73.

32. Daunes-Duport C, Pietsch T, Lantos PL. DNET pathology and genetics of tumors of the neurosurgery system. Lyon, France: LAPC Bess; 2000. p. 103-4.

33. Blumcke I, Glancke K, Wardelmann E, Beyenburg S, Kral T, Sarioglu $\mathrm{N}, \mathbb{e}$ al. The CD-34 epitope is expressed in neoplastic and malformative lesions associated with chronic focal epilepsies. Acta Neuropathol Berl 1999;97:481-90.

34. Blumcke I, Lobecis M, Wolf HK, Wiestter OD. Evidence for developmental precursor lesions in epilepsy associated glioneural tumours. Micros Res Tech 1999;46:53-8.

35. Kral T, Clusmann H, Blumcke I, Fimmers R, Ostertun B, Kurthen M, \& al. Outcome of epilepsy surgery in focal cortical dysplasia. J Neurol Neurosurg Psychiatry 2003;74:183-8.

Accepted on 12-04-2008

Source of Support: Nil, Conflict of Interest: None declared. 